\title{
Testing dynamic stabilisation in complex Langevin simulations
}

Felipe Attanasio ${ }^{1}$, Benjamin Jäger ${ }^{* 1,2}$

${ }^{1}$ Department of Physics, College of Science, Swansea University, United Kindgom

${ }^{2}$ ETH Zürich, Institute for Theoretical Physics, Wolfgang-Pauli-Str. 27, 8093 Zürich, Switzerland

E-mail: bejaeger@phys.ethz.ch

Complex Langevin methods have been successfully applied in theories that suffer from a sign problem such as QCD with a chemical potential. We present and illustrate a novel method (dynamic stabilisation) that ensures that Complex Langevin simulations stay close to the SU(3) manifold, which lead to correct and improved results in the framework of pure Yang-Mills simulations and QCD in the limit of heavy quarks.

34th annual International Symposium on Lattice Field Theory

24-30 July 2016

University of Southampton, UK

${ }^{*}$ Speaker. 


\section{Introduction}

The famous sign problem is encountered in many areas of physics. One of the most prominent examples is QCD with non-zero baryon chemical potential. The fermion determinant leads to a complex weight in the associated Euclidean path integrals, and thus prevents direct determination of the QCD phase diagram with standard Monte Carlo techniques. Complex Langevin simulations have been shown to enable simulations even when the sign problem is severe [1, 2, 3, 4, 5]. The hope is that fully dynamical QCD can be studied with this stochastic quantization, which was first introduced in $[6,7,8,9,10]$. A crucial step forward was made by employing gauge cooling [11, 12], which is used to control the distance from the unitary sub-manifold. Gauge cooling made it possible to obtain the first results in QCD, both with heavy $[13,14]$ and with lighter quarks [2, 3]. However, as already noted in [14], gauge cooling is less effective at smaller values of the gauge coupling $\beta$, i.e. on coarser lattices. While this is not a problem per se, eventually the continuum limit will have to be taken, it limits the applicability to lower temperatures, which would require very large lattices. Hence it is worthwhile to study modifications of the CL process, which ameliorate the process at smaller $\beta$ values, which will be presented in the following.

\section{Dynamic stabilisation}

A generic first-order Langevin update step in Langevin time $\theta$ can be written as [15]

$$
U_{x, v}(\theta+\varepsilon)=\exp \left[\mathrm{i} \lambda^{a}\left(-\varepsilon D_{x, v}^{a} S+\sqrt{\varepsilon} \eta_{x, v}^{a}\right)\right] U_{x, v}(\theta),
$$

where $\lambda^{a}$ are the Gell-Mann matrices and the action $S$ contains the Yang-Mills gauge part and the logarithm of the fermion determinant as

$$
S=S_{\mathrm{YM}}-\ln \operatorname{det} M .
$$

For simplicity, we skip the discussion about the logarithm of the fermion determinant and its potential problems on the convergence of the Langevin process. Detailed discussion can be found in $[16,17,18,19]$. The drift of the action, i.e. $-D_{x, v}^{a} S$, governs the dynamics of this stochastic process, which is realized by the Gaussian white noise $\eta_{x, v}^{a}$. To circumvent the complexity of the Euclidean path integral and evade the sign problem, we extend the gauge group from $\mathrm{SU}(3)$ to $\mathrm{SL}(3, \mathbb{C})$, so that all links can symbolically written as

$$
U_{x, v}=\exp \left[\mathrm{i} a \lambda^{c}\left(A_{x, v}^{c}+\mathrm{i} B_{x, v}^{c}\right)\right],
$$

where $a$ is the lattice spacing and $A_{x, v}^{c}\left(B_{x, v}^{c}\right)$ are the real (imaginary) coefficients of the Gell-Mann matrices. For $\mathrm{SU}(3)$ gauge links the imaginary components vanish completely, i.e. $B_{x, v}^{c}=0$. A measure of the distance to $\mathrm{SU}(3)$ sub-manifold is given by the unitarity norm, defined as

$$
d_{2}=\frac{1}{3 V} \sum_{x, v} \operatorname{Tr}\left(U_{x, v} U_{x, v}^{\dagger}-1\right)^{2} .
$$

The main idea of our new method, dynamic stabilisation, is to add a force to the Langevin dynamics, i.e.

$$
U_{x, v}(\theta+\varepsilon)=\exp \left[i \lambda^{a}\left(-\varepsilon D_{x, v}^{a} S+i \varepsilon \alpha_{D S} M_{x}^{a}+\sqrt{\varepsilon} \eta_{x, v}^{a}\right)\right] U_{x, v}(\theta)
$$


that formally vanishes in the continuum limit and provides a drift that is directed towards the $\mathrm{SU}(3)$ sub-manifold. A possible solution is given by [20]

$$
M_{x}^{a}=i b_{x}^{a}\left(\sum_{c} b_{x}^{c} b_{x}^{c}\right)^{3} \quad b_{x}^{a}=\operatorname{Tr}\left[\lambda^{a} \sum_{v} U_{x, v} U_{x, v}^{\dagger}\right],
$$

where the sum over $c$ is written explicitly for clarification. The additional forces are independent of the direction of the link and hence are applied equally in all four directions. We point out that this solution is not unique and many different choices can be found. We note here that the additional term in the drift is not invariant under $\operatorname{SL}(3, \mathbb{C})$ gauge transformations and not holomorphic, and hence cannot be derived from an action principle. However it is still invariant under SU(3) transformations. Instead it should be viewed as an additional contribution to the drift, whose role is to contain the dynamics in the non-compact direction. Due to the presence of this term the standard justification of Complex Langevin [21, 22] can no longer be used, since the direct connection between the drift and complex weight is jeopardised. However, an expansion in powers of the lattice spacing shows that

$$
M_{x}^{a} \sim a^{7}\left(\bar{B}_{y}^{c} \bar{B}_{y}^{c}\right)^{3} \bar{B}_{x}^{a}+\mathscr{O}\left(a^{8}\right) \quad \text { with } \quad \bar{B}_{x}^{a}=\sum_{\mu} B_{x, \mu}^{a},
$$

and hence the additional term is indeed formally irrelevant in the continuum limit. We emphasise that this is very different from gauge cooling, where the dynamics is controlled via $\operatorname{SL}(3, \mathbb{C})$ gauge transformations and not via a modification of the drift. In spite of this open theoretical issue, we will see below that the results obtained with DS are very promising.

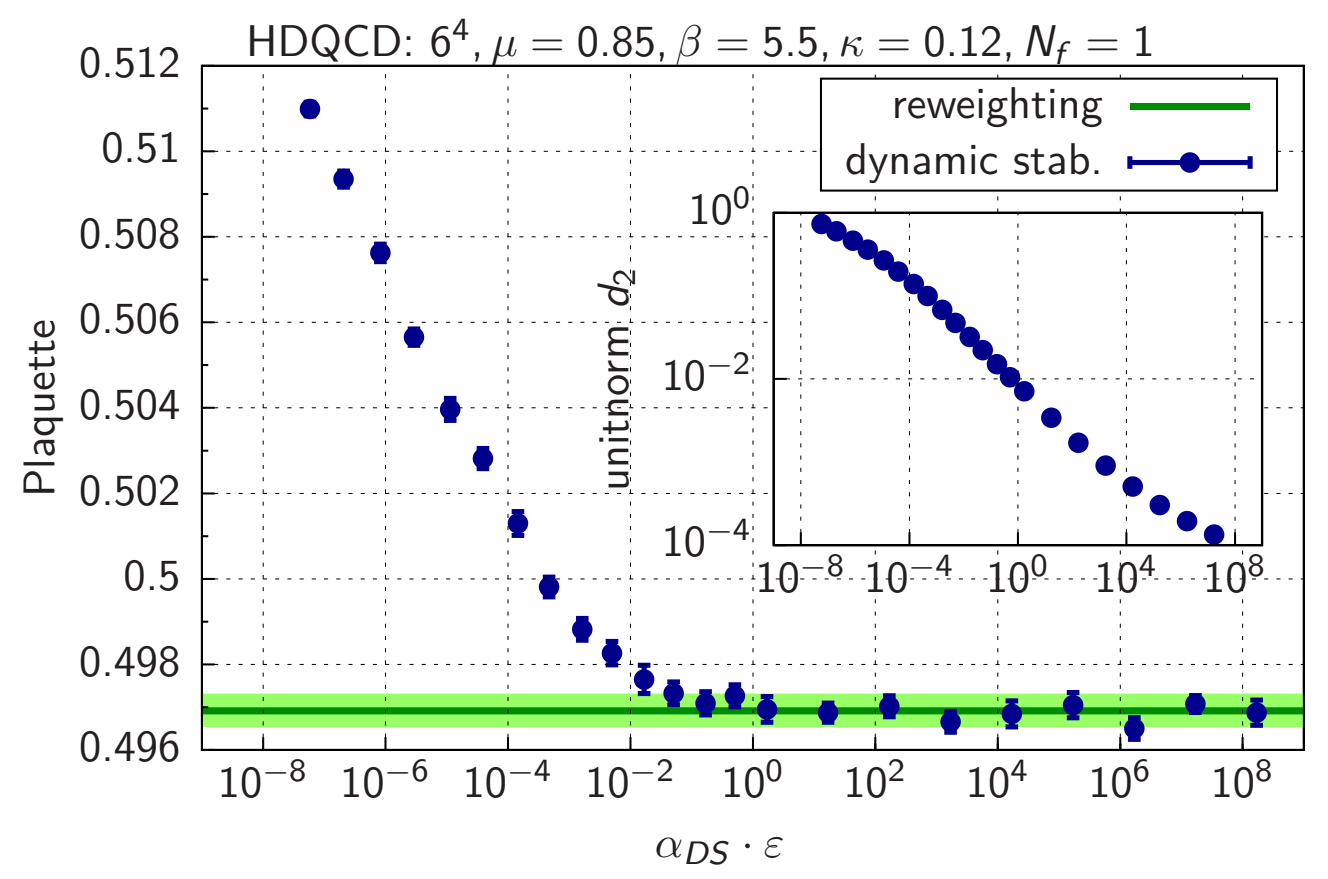

Figure 1: The plaquette as a function of the dynamic stabilisation coefficient $\alpha_{D S}$ times the average stepsize $\varepsilon$. For sufficient large values $\gtrsim 10^{0}$, we find perfect agreement with reweighting results. The unitarity norm is shown in the small sub-section of this figure as a function of the same $\alpha_{D S} \cdot \varepsilon$. 
The coefficient $\alpha_{D S}$ allows us to vary the strength of the additional force in order to tune the distance to $\mathrm{SU}(3)$. For very large values of $\alpha_{D S}$ this relates to a dynamic re-unitarization of the gauge links. In the limit of small coefficients the drift becomes irrelevant for the dynamics. The right choice of the parameter $\alpha_{D S}$ depends on the application and needs some tuning. Dynamic stabilisation can be trivially combined with gauge cooling and we found that one step of gauge cooling is very beneficial and sufficient for the overall convergence. Therefore we use a single step of gauge cooling in all simulation results shown in the following. Figure 1 shows the plaquette as a function of the dynamic stabilisation coefficient $\alpha_{D S}$ times the average stepsize $\varepsilon$ for a HDQCD simulation. For small values the results significantly deviate from reweighting results, further illustrating that the unitarity norm is too large, which is shown in the . For $\alpha_{D S} \gtrsim 10^{0}$ the plaquette is in perfect agreement with reweighting data. Please note that the force added to the drift scales with a high powers of $\bar{B}_{y}^{c}$ and thereby indirectly with unitarity norm, which is small for large combinations of $\alpha_{D S} \cdot \varepsilon$.

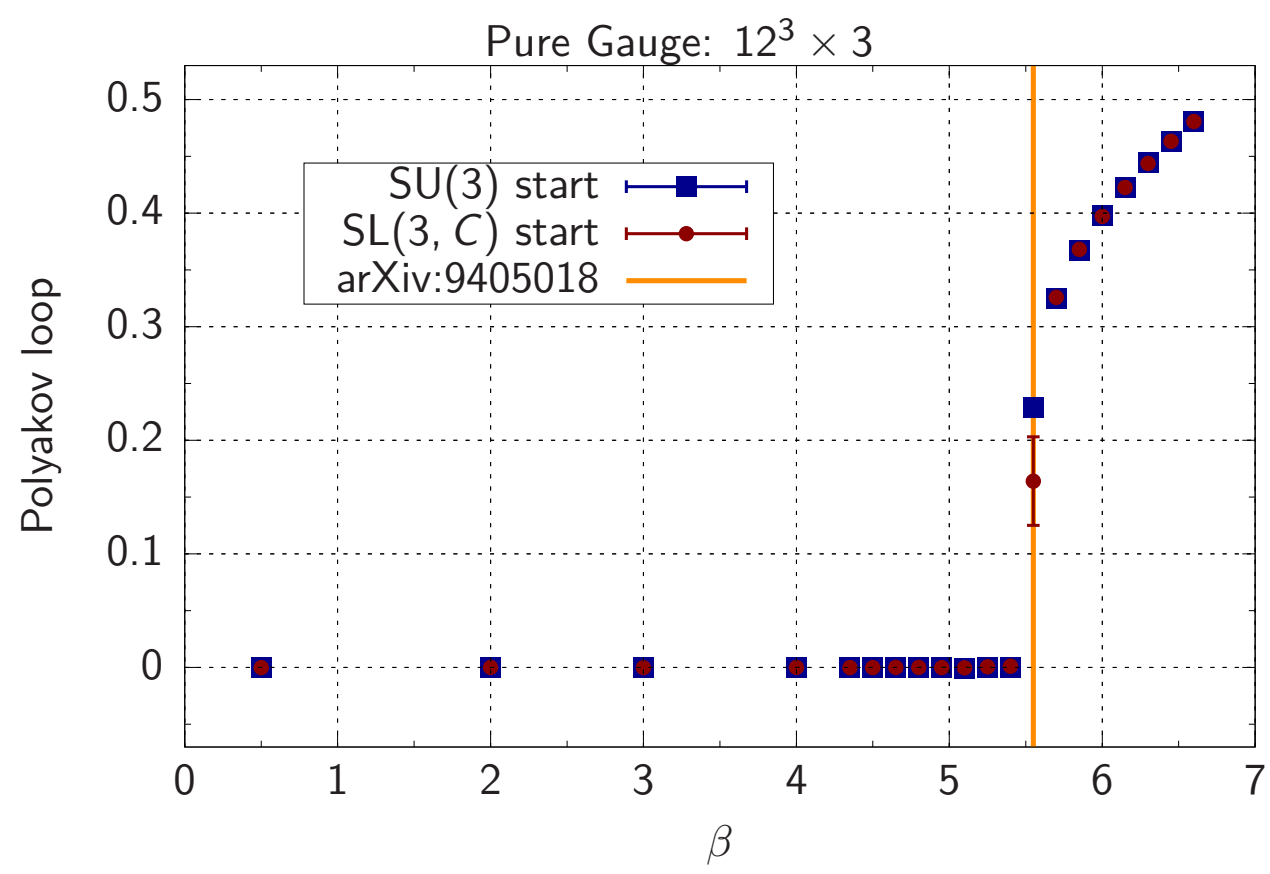

Figure 2: Pure Yang-Mills simulation using dynamic stabilisation as a function of the gauge coupling $\beta$ for two different initial conditions. The result from Hybrid Monte Carlo [23] have been added for comparison.

For pure Yang-Mills simulations, the complexification of the gauge degrees of freedom is not necessary, since the problem is free of a sign problem and standard Monte Carlo techniques can be applied in a straightforward way. However, it serves as a good testing scenario to check that complex Langevin simulations still work. By using $\operatorname{SL}(3, \mathbb{C})$ gauge links, we effectively use too many degrees of freedom and allow the system to generate imaginary drifts, which ideally would exactly vanish. However, round off errors and subsequent small imaginary components will generate non-zero $B_{x, v}^{c}$. We previously showed that complex Langevin simulations using just gauge cooling indeed reproduce the correct deconfinement transitions, when we start with an unitary configuration, i.e $B_{x, v}^{c}=0$ and use gauge cooling to maintain a sufficient small unitarity norm [24]. However, using a generic $\operatorname{SL}(3, \mathbb{C})$ start, with $B_{x, v}^{c} \neq 0$, leads to a rising unitarity norm along 
the Langevin time until the simulations fail. Adding dynamic stabilisation correctly reproduce the deconfinement transition as obtained in [23]. Figure 2 shows such a comparison of pure YangMills simulation using complex Langevin with two different initial conditions, one using purely $\mathrm{SU}(3)$ links and the other uses a full $\mathrm{SL}(3, \mathbb{C})$ configuration. The known result, shown as a yellow line in Figure 2, is correctly reproduced. It is worth mentioning that in case of dynamic stabilisation using a $\operatorname{SL}(3, \mathbb{C})$ start, the unitarity norm remains small but finite, implying that $B_{x, v}^{c} \neq 0$.

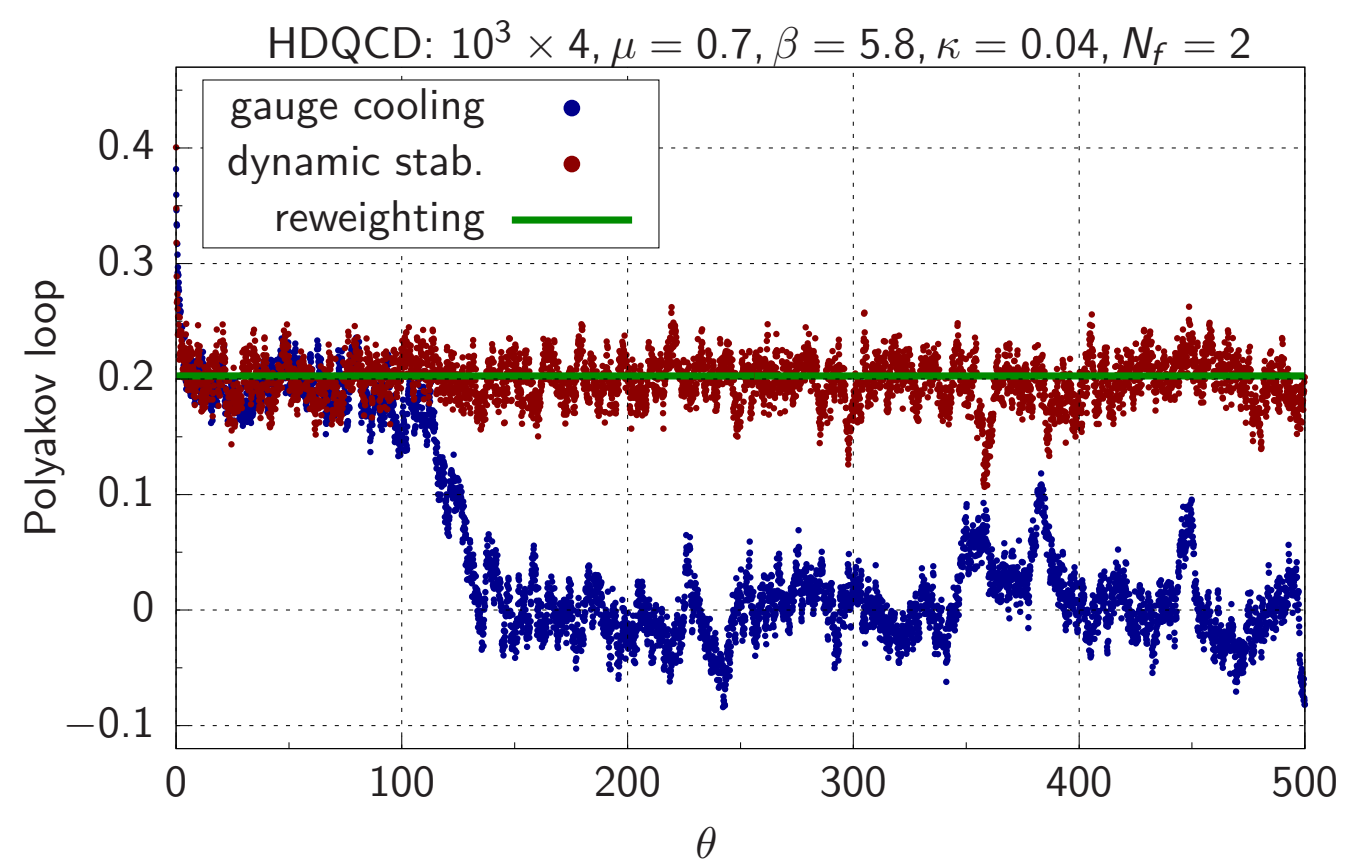

Figure 3: Polyakov loop as a function of Langevin time $\theta$. The simulation using just gauge cooling (blue) changes to a wrong result at $\theta \sim 100$. The one including dynamical stabilisation (red) remains at the correct value indicated by reweighting (green).

For QCD in the limit of heavy quarks (HDQCD), we have seen examples [14], in which complex Langevin simulations diverted away after an arbitrary Langevin time from the expected results. The change of behaviour coincides with the unitarity norm exceeding $\mathscr{O}(0.5)$. An example is shown in Figure 3 in blue. The simulation parameters are listed at the top of the plot. After adding the dynamic stabilisation force to Langevin dynamics we find agreement with reweighting for all Langevin time. Different gauge couplings are shown in Figure 4. We find significant deviations between gauge cooling and reweighting results, taken from [11], using adaptive gauge cooling over 500 Langevin time for $\beta \lesssim 5$.8. Dynamic stabilisation improves the convergence of HDQCD simulations and enable simulations for small gauge couplings. Further test on different models and scenarios are subject to future studies.

\section{Conclusions and Outlook}

We presented here two tests of our newly proposed method of dynamic stabilisation for pure gauge simulation and the heavy dense approximation of QCD (HDQCD). In both cases we have seen clear improvements over gauge cooling and very good agreement with reweighting. Further 


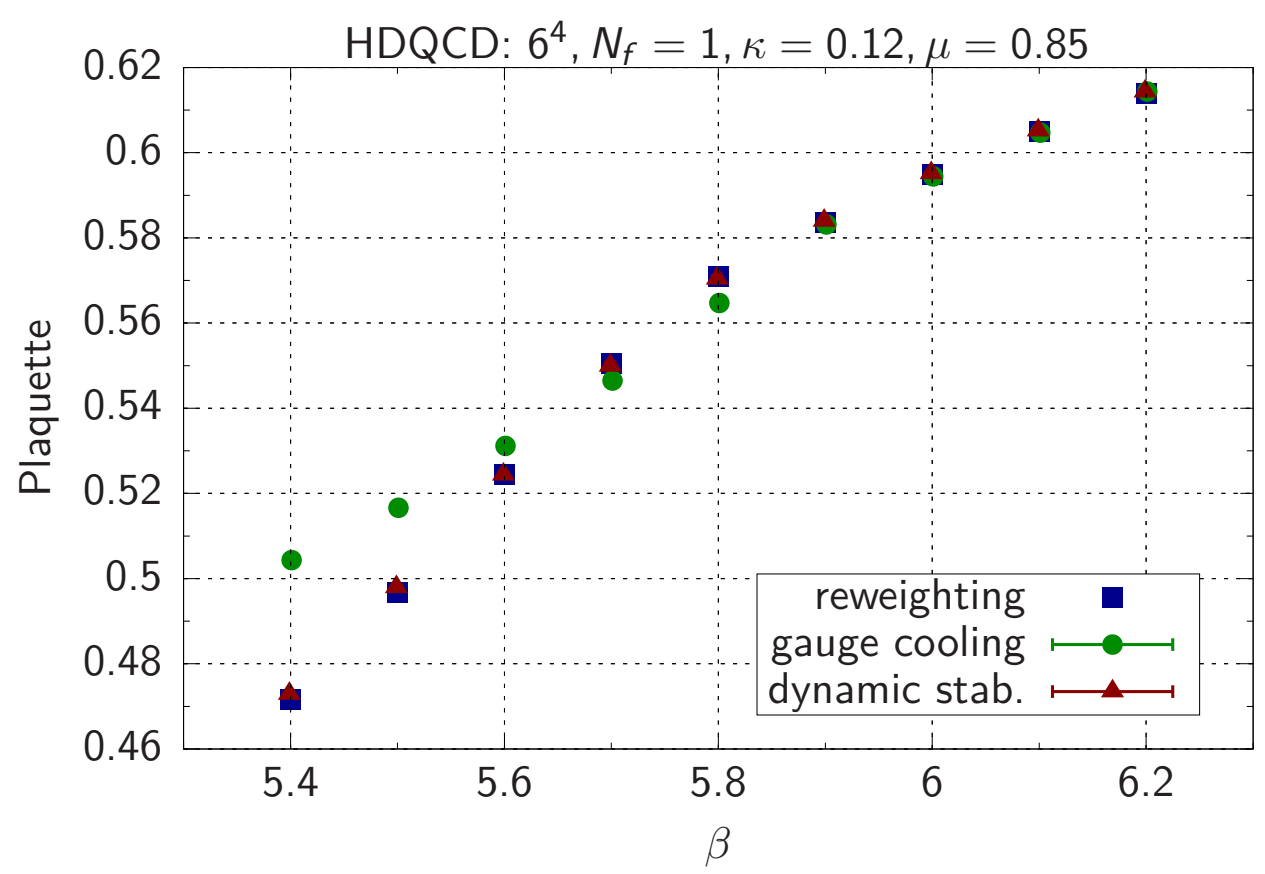

Figure 4: The plaquette as a function of the gauge coupling $\beta$. At small gauge couplings, gauge cooling, using an adaptive number (up to 20) of cooling steps, shows significant deviations from reweighting data taken from [11]. Including dynamic stabilisation improves convergence for all available $\beta$.

tests will validate if this method is suitable for simulation of dynamical QCD. Here in particular smaller gauge couplings allow simulations in the confined phase and might enable simulations at low temperatures. The method, however, remains heuristic and requires adequate testing and justification.

Acknowledgements: The authors thank Gert Aarts, Dénes Sexty, Erhard Seiler and IonOlimpiu Stamatescu for valuable discussions and collaboration. We also appreciate the reweighting results obtained and provided by Dénes Sexty and Ion-Olimpiu Stamatescu. We are grateful for the computing resources made available by HPC Wales. We acknowledge the STFC grant ST/L000369/1, the Royal Society and the Wolfson Foundation. FA is grateful for the support through the Brazilian government programme "Science without Borders" under scholarship number BEX 9463/13-5.

\section{References}

[1] G. Aarts and I.-O. Stamatescu, Stochastic quantization at finite chemical potential, JHEP 09 (2008) 018, [0807.1597].

[2] D. Sexty, Simulating full QCD at nonzero density using the complex Langevin equation, Phys. Lett. B729 (2014) 108-111, [1307 . 7748].

[3] G. Aarts, E. Seiler, D. Sexty and I.-O. Stamatescu, Simulating QCD at nonzero baryon density to all orders in the hopping parameter expansion, Phys. Rev. D90 (2014) 114505, [1408.3770]. 
[4] D. K. Sinclair and J. B. Kogut, Exploring Complex-Langevin Methods for Finite-Density QCD, PoS LATTICE2015 (2016) 153, [1510.06367].

[5] K. Nagata, J. Nishimura and S. Shimasaki, The argument for justification of the complex Langevin method and the condition for correct convergence, 1606.07627.

[6] G. Parisi and Y.-s. Wu, Perturbation Theory Without Gauge Fixing, Sci. Sin. 24 (1981) 483.

[7] G. Parisi, On Complex Probabilities, Phys. Lett. B131 (1983) 393-395.

[8] J. R. Klauder, Stochastic Quantization, Acta Phys. Austriaca Suppl. 25 (1983) 251-281.

[9] J. R. Klauder, A Langevin Approach to Fermion and Quantum Spin Correlation Functions, J. Phys. A16 (1983) L317.

[10] J. R. Klauder, Coherent State Langevin Equations for Canonical Quantum Systems With Applications to the Quantized Hall Effect, Phys. Rev. A29 (1984) 2036-2047.

[11] E. Seiler, D. Sexty and I.-O. Stamatescu, Gauge cooling in complex Langevin for QCD with heavy quarks, Phys. Lett. B723 (2013) 213-216, [1211. 3709].

[12] G. Aarts, L. Bongiovanni, E. Seiler, D. Sexty and I.-O. Stamatescu, Controlling complex Langevin dynamics at finite density, Eur. Phys. J. A49 (2013) 89, [1303.6425].

[13] G. Aarts, F. Attanasio, B. Jäger, E. Seiler, D. Sexty and I.-O. Stamatescu, The phase diagram of heavy dense QCD with complex Langevin simulations, Acta Phys. Polon. Supp. 8 (2015) 405, [1506.02547].

[14] G. Aarts, F. Attanasio, B. Jäger and D. Sexty, The QCD phase diagram in the limit of heavy quarks using complex Langevin dynamics, JHEP 09 (2016) 087, [1606. 05561 ].

[15] P. H. Damgaard and H. Hüffel, Stochastic Quantization, Phys. Rept. 152 (1987) 227.

[16] A. Mollgaard and K. Splittorff, Complex Langevin Dynamics for chiral Random Matrix Theory, Phys. Rev. D88 (2013) 116007, [1309.4335].

[17] K. Splittorff, Dirac spectrum in complex Langevin simulations of QCD, Phys. Rev. D91 (2015) 034507, [1412.0502].

[18] J. Nishimura and S. Shimasaki, New Insights into the Problem with a Singular Drift Term in the Complex Langevin Method, Phys. Rev. D92 (2015) 011501, [1504 . 08359].

[19] J. Greensite, Comparison of complex Langevin and mean field methods applied to effective Polyakov line models, Phys. Rev. D90 (2014) 114507, [1406.4558].

[20] G. Aarts, F. Attanasio, B. Jäger and D. Sexty, Complex Langevin in Lattice QCD: dynamic stabilisation and the phase diagram, Acta Phys. Polon. Supp. 9 (2016) 621, [1607.05642].

[21] G. Aarts, E. Seiler and I.-O. Stamatescu, The Complex Langevin method: When can it be trusted?, Phys. Rev. D81 (2010) 054508, [0912.3360].

[22] G. Aarts, F. A. James, E. Seiler and I.-O. Stamatescu, Complex Langevin: Etiology and Diagnostics of its Main Problem, Eur. Phys. J. C71 (2011) 1756, [1101.3270].

[23] G. Cella, G. Curci, A. Vicere and B. Vigna, The SU(3) deconfining phase transition with Symanzik action, Phys. Lett. B333 (1994) 457-460, [hep-lat/ 9405018 ].

[24] G. Aarts, F. Attanasio, B. Jäger, E. Seiler, D. Sexty and I.-O. Stamatescu, Towards the heavy dense QCD phase diagram using Complex Langevin simulations, PoS LATTICE2015 (2016) 154, [1510.09098]. 